\title{
Robust and miniaturized non-incremental fiber-optic distance sensor for turbo machine rotor deformation and vibration monitoring
}

\author{
Dreier, Florian; Pfister, Thorsten; Czarske, Jürgen \\ TU Dresden, Institut f. Grundlagen der Elektrotechnik und Elektronik, Professur f. Mess- und Prüftechnik \\ Helmholtzstraße 18, 01062 Dresden, Germany
}

\begin{abstract}
In order to improve the safety, the lifetime and the energy efficiency of turbo machines, the dynamic behaviour of the rotor has to be analysed and optimized. Thus, rotor unbalances, dynamic deformations and blade vibrations as well as blade tip clearance changes have to be monitored during operation to optimize the rotor design and to validate numerical models. However, these are big challenges for metrology, since small, robust and non-contact measurement techniques are required offering both micron accuracy and microsecond temporal resolution.

We will give a thorough description of a robust, non-incremental fiber-optic distance sensor, which is only $20 \mathrm{~mm}$ in diameter and less than $100 \mathrm{~mm}$ in length. Simulative results of its numerical optimization using the software tool ZEMAX are presented. In addition, simulated data is compared with laboratory measurements to confirm the reliability of the simulation.
\end{abstract}

\section{Introduction}

Simulations were carried out to investigate the behaviour of fast rotating rotors in turbo machines, but the results are insufficient, especially at innovative materials, such as fiber reinforced materials. Hence, adequate measurement systems are necessary to investigate the dynamic behaviour during operation of bladed rotors in turbo machines. Since the blades reach high tangential velocities up to several hundred meters per second, fast, contactless and precise measurement methods are necessary.

Capacitive and inductive probes are applied to measure blade tip clearance in process, but they are influenced by electro-magnetic disturbances and need time-consuming in-situ calibrations. In addition, they rely on electrical conducting materials and, thus, fail at innovative materials such as fiber reinforced materials. Optical measurement methods overcome this drawback, but for most of them the measurement rate is restricted to some $\mathrm{kHz}$ either by the speed of mechanical scanning (optical coherence tomography [1]) or by the detector frame rate and minimum exposure time (triangulation). This leads to an increasing measurement uncertainty with increasing tip speed of the blades and, above a certain threshold, measurements are no longer possible during a single blade passage [2].

Scanning laser vibrometry is commonly used for vibration and deformation investigations in laboratory test rigs at low rotational speed, but it fails at in-situ measurements at turbo machines due to the necessity of a large optical access in axial direction [3]. In-situ vibration measurements are carried out using strain gauges. However the durability, the application and the signal transmission is challenging due to the high rotational speed [4,5]. Blade tip timing also allows in-situ measurements, but it is limited to tangential vibrations and needs often previous knowledge to determine unambiguous vibration frequencies, because the signals are heavily aliased [5].

In this contribution, we introduce a recently developed non-incremental laser Doppler distance sensor (LDDS) employing two superposed fan-shaped interference fringe systems with contrary fringe spacing gradients. Via the resulting two independent measuring channels, it measures simultaneously the transverse object velocity and the radial position of rotating objects with micron precision and high temporal resolution concurrently. The outstanding feature of the LDDS is that its measurement uncertainty is generally independent of the object velocity, which enables precise deformation and vibration measurements also at high rotor speed [6]. Moreover, this sensor does not require an in situ calibration and it allows measuring blade tip clearance as well as radial and lateral rotor vibrations simultaneously. Employing a rugged LDDS prototype with integrated water cooling, successful test measurements were accomplished at a transonic centrifugal compressor as well as at a vacuum highspeed rotor test rig $[7,8]$. Based on these results, a noval miniaturized concept for the sensor setup will be presented, which is robust and thermostable and, thus, enables in-situ blade vibration and tip clearance measurements even at high ambient temperatures. 


\section{Sensor principle}

The laser-Doppler distance sensor is based on a conventional laser Doppler velocimeter which measures the transverse velocity $v$ of scattering particles. Two coherent laser beams are superposed and, hence, create an interference fringe system with a defined fringe spacing $d$, which corresponds with the measurement volume. The mean fringe spacing depends on the half crossing angel $\Phi$ of the two laser beams and the wavelength $\lambda$ of the laser light:

$$
d=\frac{\lambda}{2 \sin (\phi)} .
$$

A scattering object crossing the interference fringe system generates an amplitude modulated scattered light signal, which can be detected by a photodetector. By evaluating the modulation frequency $f$, also known as Doppler frequency, and the interference fringe spacing $d$ known from a proper calibration, the object velocity $v$ can be calculated by means of

$$
v_{x}=d \cdot f .
$$

The novel laser-Doppler distance sensor was developed to obtain the transverse object velocity $v_{x}$ and the axial position $z$ of the measuring object simultaneously (see figure 1) [3]. Therefore two fan-shaped interference fringe systems with different gradients of the fringe spacing $\partial d_{i}(z) / \partial z, i=1,2$ are superposed at the same place. Hereby, the waist of the Gaussian beam placed in front of the measurement volume results in a divergent interference fringe system. For the second, convergent interference fringe system, the waist of the beam is set behind the measurement volume.

A physical separation of the two interference fringe system can be achieved by applying wavelength division multiplex, frequency division multiplex or time division multiplex. Hence, two Doppler frequencies $f_{1,2}$ can be measured, whereas the quotient of these two Doppler frequencies forms an unambiguous calibration quotient $q$ which depends on the axial position of the scattering particle, but not on the object velocity $v$ in $x$-direction.

$$
q(z)=\frac{f_{2}(v, z)}{f_{1}(v, z)}=\frac{v(z) / d_{2}(z)}{v(z) / d_{1}(z)}=\frac{d_{1}(z)}{d_{2}(z)}
$$

Hence, a proper calibration procedure can be determined to derive the axial position $z$ as well as the object velocity $v_{x}$ of the scattering object (see figure (1)).

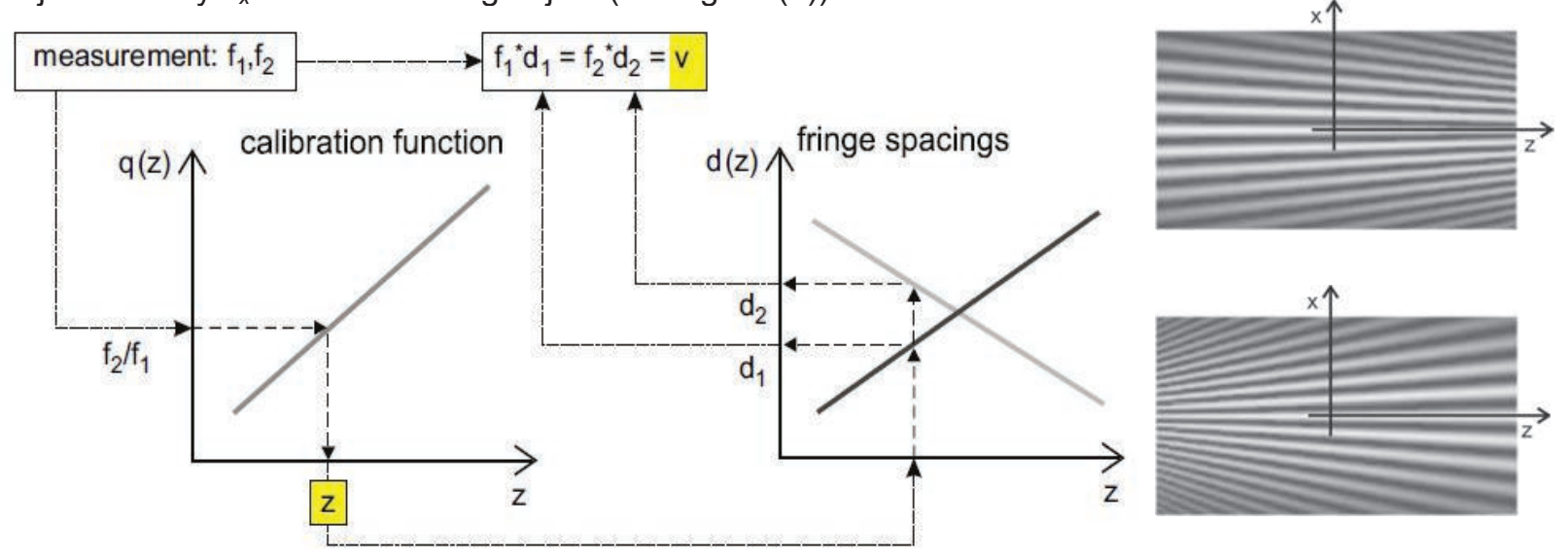

Fig. 1: Sensor principle for simultaneous determination of the position $z$ and the object velocity $v_{x}$ (left) using a divergent and a convergent interference fringe system (right).

\section{Concept for the thermostable, miniaturized and robust sensor head}

In-situ measurements at turbo machines imply rough ambient conditions, such as high temperatures up to $1000{ }^{\circ} \mathrm{C}$ and some hundred bars of pressure. Since the sensor needs an optical access to the blades of the rotor, it is advisable to mount it into the housing of the turbo machine. Thus, the sensor is directly exposed to the rough ambient conditions, which predominate inside the turbo machine. Normally, a drill hole serves as the optical access for the sensor, which has to be small in size to reduce the disturbance to the functionality of the turbo machine. Due to the fact that the senor is integrated in the housing of the turbo machine, it has to withstand vibrations, which occur at in-situ measurements at turbo machines. Considering all these aspects, a miniaturized, robust and thermostable sensor is necessary. 
These considerations lead to modular setup, since all active components has to be spatially separated from the rough conditions at the turbo machine. All in all, three modules can be identified, i.e. the light sending unit, the sensor head and the scattered light detection unit, which are connected to each other with thermostable fibers. Thus, only the sensor head, which forms the measurement volume and detects the backscattered light, has to withstand the rough ambient conditions.

One possible sensor head concept is well-known from laser-Doppler velocimetry and consists of four light-sending fibers for the four partial beams of the two interference fringe systems (see figure 2). Every partial beam is collimated separately and directed to the common crossing point, which forms the measurement volume. Another fiber is necessary to guide the scattered light to the detection unit, so that all in all five thermostable fibers have to be connected to the sensor head. The miniaturization potential of this concept is limited, because every beam needs a separate collimating optic and additional elements to adjust the beam propagation direction and the waist position in the measurement volume. Furthermore, mechanical disturbances, such as changing temperature conditions, could unequally effect the different beams, which leads to no common crossing point of the partial beams and therefore make, in worst case, measurements impossible. Hence, the miniaturization potential of this concept is limited to the mechanical complexity of the setup to assure the robustness against the rough ambient conditions.
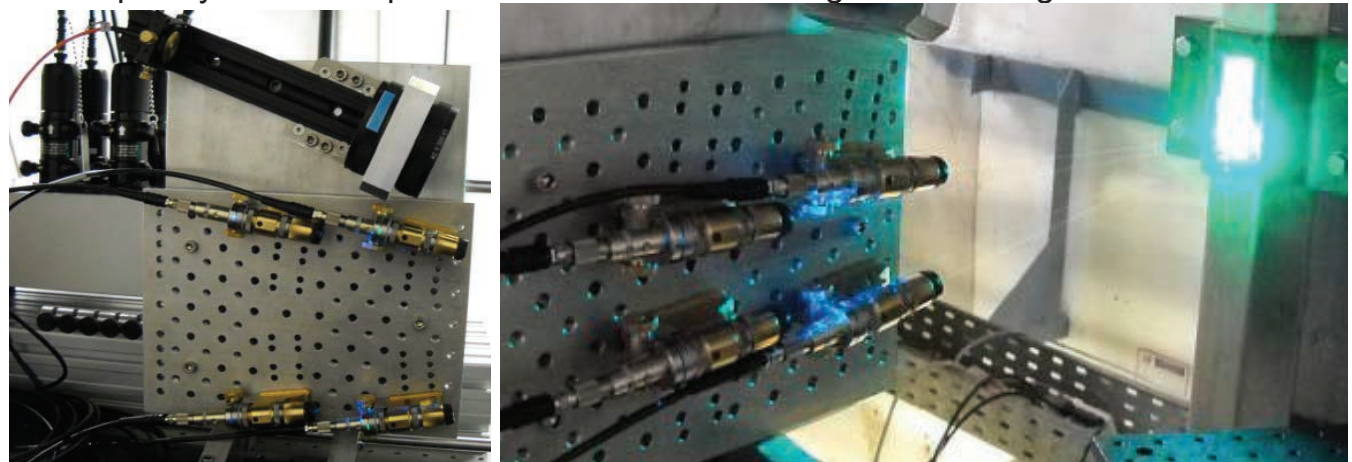

Fig. 2: Fiber optical sensor head with 5 separated optics for the four sending beams and the scattered light detection.

Another sensor head concept has been tested successfully at a radial compressor using wavelengthdivision multiplex. In this case, the light sending unit consists of two fiber-coupled transversal singlemode laser diodes with different wavelength, which are coupled into one singlemode fiber using a $2 \times 1$ fiber coupler. The fiber is connected to the sensor head where the light field is imaged on a transmission phase grating using a Keplerian telescope. The +1 . and the -1 . diffraction order of the grating is used to generate the interference fringe system by imaging and superposing these orders via a second telescope to the measurement volume (see figure 3). In this concept a special diffractive lens (DOE) is used to achieve a fixed spatial separation of the waist positions of the two different wavelengths. This is possible due to the characteristic high dispersion of the DOE, which is about 30 times higher in comparison to refractive lenses.

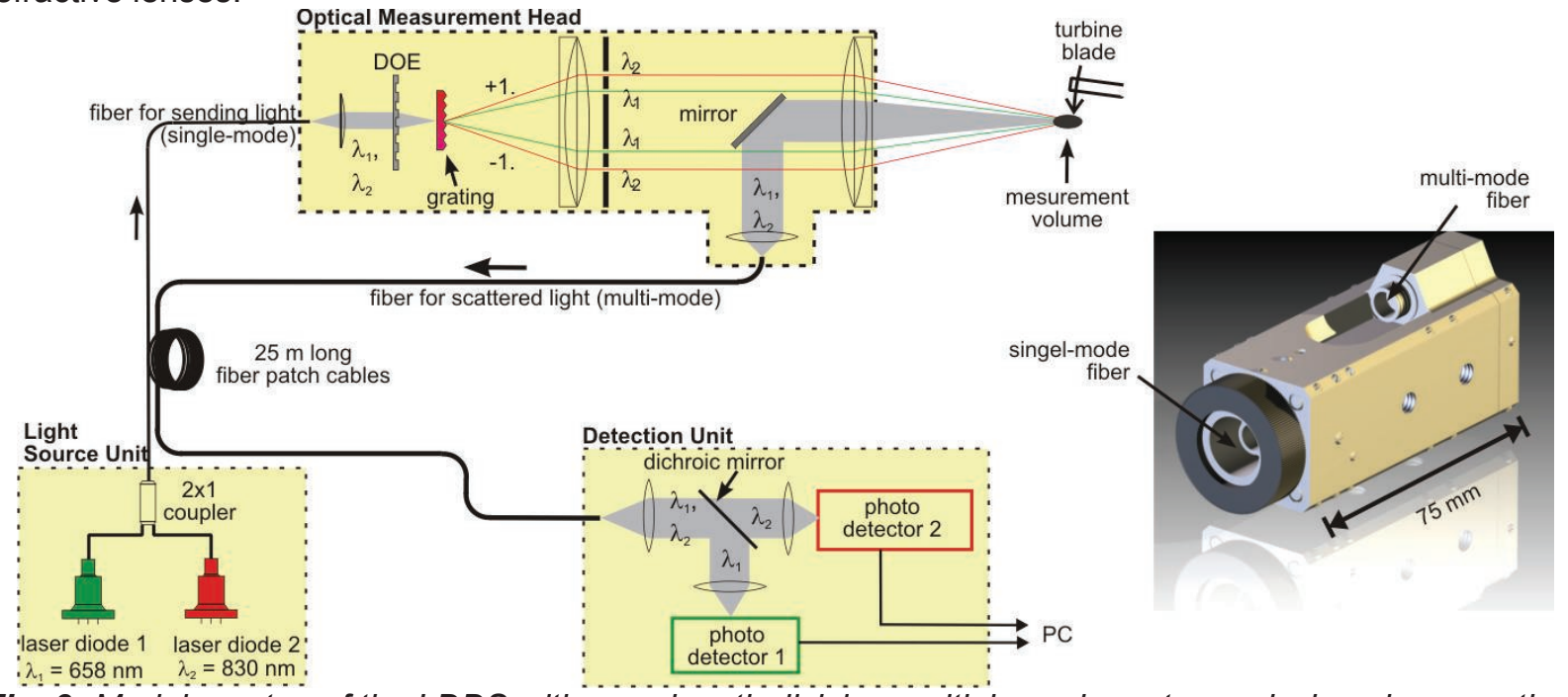

Fig. 3: Modular setup of the LDDS with wavelength division multiplex using a transmission phase grating for beam splitting and a DOE for separation of the waist positions (left). According miniaturized sensor head (right). 
The scattered light is detected in backward direction using the aperture between the partial beams. By using a transmission grating for splitting the beams, this concept is in general more robust than the first one, because mechanical disturbances influence all partial beams equally. Thus, a wavelength independent common crossing point can be guaranteed if achromatic lenses are used for the second Keplerian telescope. One drawback is the low potential for miniaturisation, because a large amount of optical components is needed for this concept. In addition, a thermostable setup is hardly achievable, because the achromatic lenses are in principle made of two different glass types with different refractivities to correct the chromatic aberration. But fused silica is the only mineral glass which withstands temperatures of more than $1000^{\circ} \mathrm{C}$ and consequently achromatic lenses cannot be designed for this temperature ranges.

The final concept is based on the modular setup and wavelength division multiplex as explained in the previous paragraph and, thus, only two thermostable fibers are necessary, one for the sending and one for the backscattered light. In contrast to the previous concept, this setup needs only one dispersive lens, which achieves both the separation of the waist positions from the measurement volume and the focusing of the laser beams. Once more, a transmission phase grating is used as beam splitter and another optical element with two further grating allows the superposition of the +1 . and the -1 . order of the beam splitting grating in the measurement volume. The scattered light is detected in backward direction by focusing the scattered light to the thermostable multimode fiber with the same lens. A wedge prism achieves a displacement of the scattered light spot in respect to the optical axis and thus, a multimode fiber can be placed next to the sending fiber to collect the scattered light. This wedge prism has a drill hole in its centre, which is necessary to avoid an interaction to the sending light field (see figure 4).

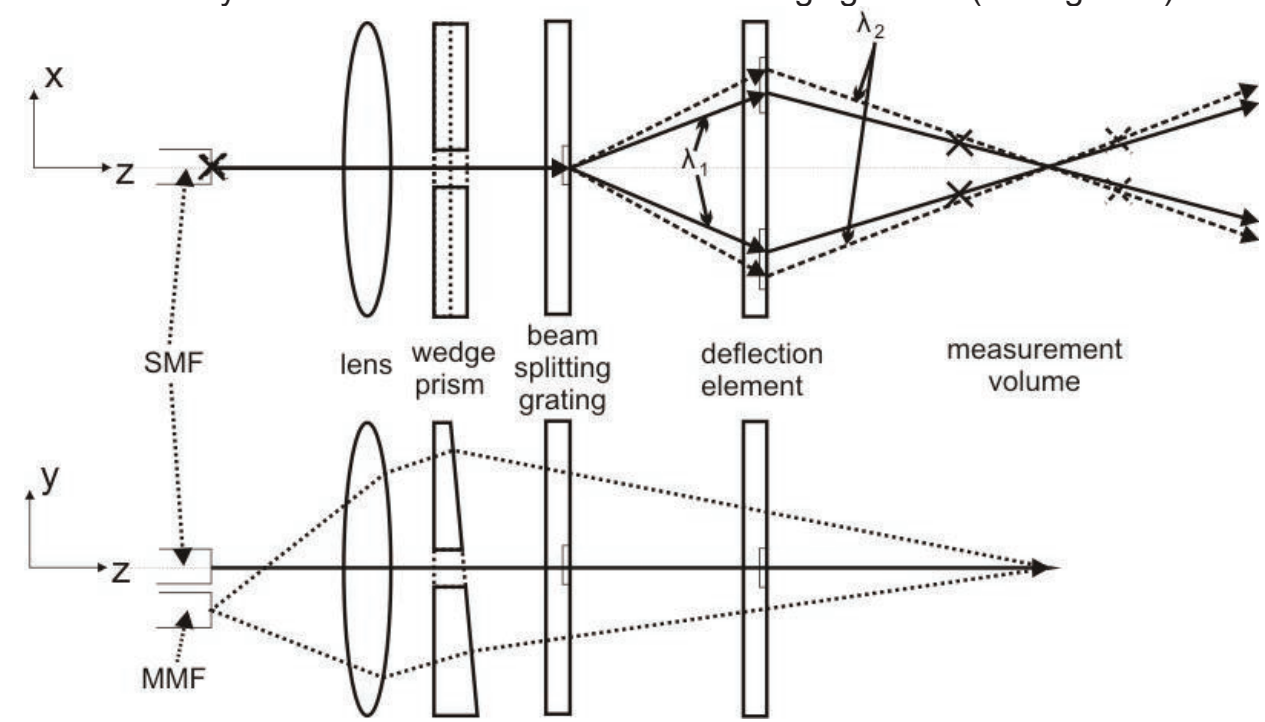

Fig. 4: Scheme of the miniaturized sensor head: The light path for sending light outgoing from a singlemode fiber (SMF) and the waist position in dependence of the wavelength (top). The back scattered light is displaced and coupled into the multimode fiber (MMF) using a wedge prism and the dispersive lens (bottom).

Since the amount of optical components is strongly reduced, a robust and miniaturized sensor head can be designed. Additionally, all optical elements as well as the fibers can be made of fused silica, so that high temperatures do not limit the functionality of the sensor. In conclusion, this concept permits operation at very high temperatures using adequate materials for the sensor housing, fused silica for the optical elements and thermostable fibers for the connection to the light sending unit and to the light detection unit respectively.

\section{Simulative investigation of the sensors properties}

The software tool ZEMAX was used for reliable investigation and numerical optimization of the expected measurement properties, such as the measurement uncertainty and the measuring length. Besides, the optimal distances between the individual optical components and the effect of misalignment of these components were determined simulatively.

In order to validate the simulated results using ZEMAX, measurements were carried out and compared with the results of the simulation. Therefore, the modular setup employing wavelength division multiplex in combination with a dispersive lens and two Keplerian telescopes was used (see section 3). Both, the 
calibration function and the fringe spacing functions are in good agreement with the results obtained by simulation (see figure 5, left). In a second step, the distance between the fiber and the collimating lens was stepwisely changed and the position of the Gaussian beam waist was observed in dependence of this parameter (see figure 5, right). The comparison shows a good agreement and, thus, the simulation with ZEMAX can either be used to optimize the sensor setup or collect the necessary tolerances for designing the thermostable housing for the sensor head.
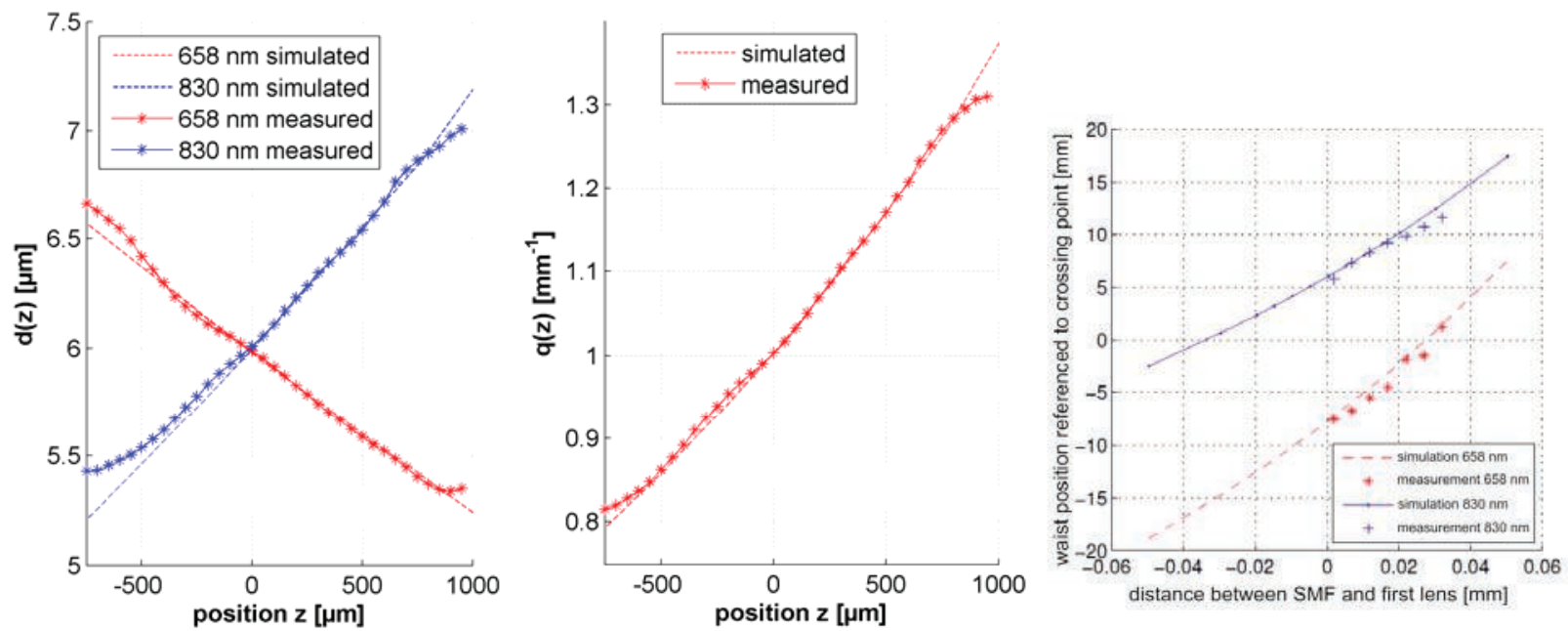

Fig. 5: Comparison of the results between measurement and simulation: fringe spacing functions (left), calibration function (middle) and the tolerance of the most critical distance, namely the distance between SMF and collimating lens (right).

Due to the validated simulation results, the novel concept of the LDDS was optimized using the software tool ZEMAX. The simulative results promise a measurement length of $2.5 \mathrm{~mm}$ and a maximal spatial resolution of about $3.5 \mu \mathrm{m}$. The fabrication tolerances of the distances between the optical elements were determined simulatively and considered for the conception of the thermostable and robust housing of the measurement head.

Since ZEMAX is capable of simulating different ambient conditions, simulations were carried out to quantify the effect of high temperatures. Therefore, ZEMAX accounts for the change of the diffractive index and the change of the geometry due to thermal expansion. For a first try a catalogue aspheric lens was used, which is made of $\mathrm{H}$-LAK54 and withstands temperatures up to $630^{\circ} \mathrm{C}$. Due to its high coefficient of thermal expansion, the calibration function is affected by the ambient temperature (see figure 6).
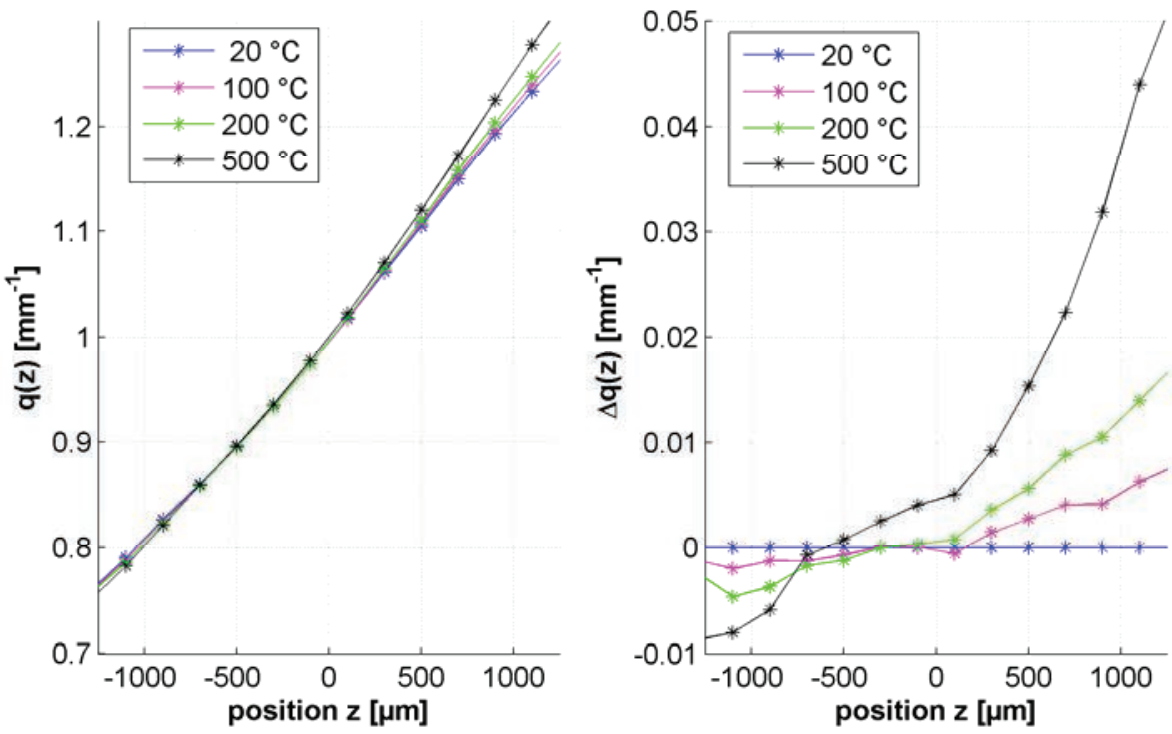

Fig. 6: Effect of high ambient temperatures to the calibration function 
At $500{ }^{\circ} \mathrm{C}$ a maximal difference of the calibration coefficient to the calibration coefficient at room temperature is about 0.05 causing a systematic measurement uncertainty of the position of about $200 \mu \mathrm{m}$. The gratings are responsible for the location of the crossing point of the partial beams, which coincides with the measurement volume. Since they are thin, plane and made of fused silica with an expansion coefficient about one magnitude smaller than H-LAK54, the location of the measurement volume nearly maintains at the same axial position. The dominant thermal effect is the change of the geometry of the used aspheric lens, such as the curvature of the lens surfaces, the thickness and the diameter. These effects directly affect the focussing as well as the waist positions of the partial beams and hence, the shape of the calibration function. To avoid the thermal dependency of the measurement result, the sensor has to be calibrated in dependence of the temperature.

Alternatively, replacing the catalogue lens with a special aspheric lens made of fused silica could reduce the changes of the lens geometry due to temperature changes. Subsequently, thermal change of the calibration function can be drastically reduced and, additionally, ambient temperatures up to approximately $1200^{\circ} \mathrm{C}$ can be reached [9]. However, for first measurement results, a catalogue lens is used, which can be subsequently exchanged with an aspheric lens made of fused silica.

\section{Summary and conclusion}

In this contribution, we introduce the simulative results of a novel miniaturized, robust and nonincremental fiber-optic sensor setup employing diffractive micro-optics in combination with a special dispersion management scheme. The sensor head is only $20 \mathrm{~mm}$ in diameter and less than $100 \mathrm{~mm}$ in length. A thermostable housing in combination with special optical components allow measurements at high ambient temperatures up to $600^{\circ} \mathrm{C}$ without active cooling which is crucial for application inside turbo machines. However, temperatures up to $1000^{\circ} \mathrm{C}$ can be reached by replacing the aspheric lens with a special lens made of fused silica. In contrast to conventional distance sensors, the LDDS offers high temporal as well as high spatial resolution and the measurement uncertainty is in principle independent of the object velocity.

Concluding these aspects, the novel concept has potential to overcome the problems of conventional sensors for in-situ measurements at turbo machines, since the LDDS measures the blade tip clearance as well as the tangential and the radial vibrations simultaneously with high precision.

\section{Acknowledgements}

The financial support from the Deutsche Forschungsgemeinschaft (funding code: $\mathrm{Cz} 55 / 23$ ) is gratefully acknowledged.

\section{Literature}

[1] A. Kempe, S. Schlamp,T. Rösgen, Low-coherence interferometric tip-clearance probe, Opt. Lett. 28, 1323-5, 2003

[2] R. G. Dorsch, G. Häusler and J. M. Herrmann, Laser triangulation: fundamental uncertainty in distance measurement, Applied Optics 33, 1306-1314, 1994.

[3] B.J. Halkon, S.J. Rothberg, Vibration measurements using continuous scanning laser vibrometry: Advanced aspects in rotor applications, Mechanical Systems and Signal Processing, 20, 1286-1299, 2006

[4] M. Zielinski, G. Ziller, Noncontact vibration measurements on compressor rotor blades, Meas. Sci. Technol. 11, S. 847-856, 2000

[5] D. Knappett, J. Garcia, „Blade tip timing and strain gauge correlation on compressor blades“, Proc. IMechE Part G: J. Aerospace Engineering, Vol. 222(4), S. 497-506, 2008

[6] T. Pfister, L. Büttner, J. Czarske, Laser Doppler profile sensor with sub-micrometre position resolution for velocity and absolute radius measurements of rotating objects, Meas. Sci. Technol. 16, S. 627-641, 2005

[7] T. Pfister, L. Büttner, J. Czarske, H. Krain, R. Schodl. Turbo machine tip clearence and vibration measurements using a fibre optic laser Doppler position sensor. Meas. Sci. Technol., 17:1693-1705, 2006

[8] P. Günther, F. Dreier, T. Pfister, J. Czarske, T. Haupt, W. Hufenbach, Measurement of radial expansion and tumbling motion of a high-speed rotor using an optical sensor system, Mechanical Systems and Signal Processing, doi:10.1016/j.ymssp.2010.08.005, 2010.

[9] LITHOSIL $®:$ Synthetic Fused Silica, Schott AG, 2010 